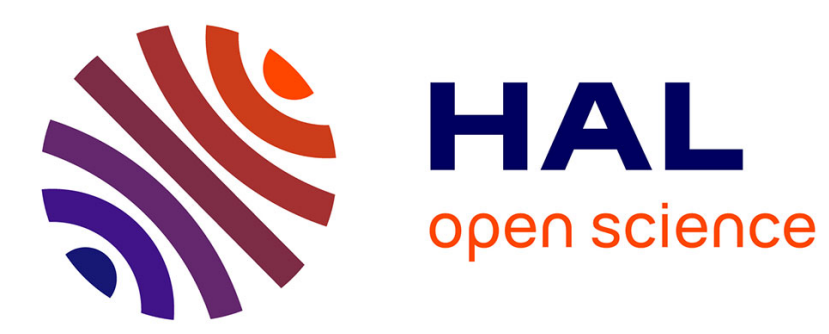

\title{
Three-dimensionality of sand ripples under a laminar shear flow
}

Vincent Langlois, Alexandre Valance

\section{To cite this version:}

Vincent Langlois, Alexandre Valance. Three-dimensionality of sand ripples under a laminar shear flow. Journal of Geophysical Research, 2005, 110, pp.F04S09. 10.1029/2004JF000278 . hal-00399954

\section{HAL Id: hal-00399954 \\ https://hal.science/hal-00399954}

Submitted on 18 Feb 2021

HAL is a multi-disciplinary open access archive for the deposit and dissemination of scientific research documents, whether they are published or not. The documents may come from teaching and research institutions in France or abroad, or from public or private research centers.
L'archive ouverte pluridisciplinaire HAL, est destinée au dépôt et à la diffusion de documents scientifiques de niveau recherche, publiés ou non, émanant des établissements d'enseignement et de recherche français ou étrangers, des laboratoires publics ou privés. 


\title{
Three-dimensionality of sand ripples under steady laminar shear flow
}

\author{
V. Langlois and A. Valance \\ Groupe Matière Condensée et Matériaux, Centre National de la Recherche Scientifique, Université de Rennes, Rennes, \\ France
}

Received 20 December 2004; revised 1 April 2005; accepted 11 May 2005; published 11 October 2005.

[1] We investigate the formation of three-dimensional sand patterns under a steady laminar shear flow using a process-based stability approach. The hydrodynamics of the problem is solved in the infinite depth case and in the long-wavelength approximation. The sand transport is described using two different models. The first one is based on a semiempirical law in terms of both the local bed shear stress and the local bed slope. The second one accounts for an additional mechanism depending on grain inertia. A three-dimensional linear stability analysis reveals that within both models, the most unstable mode is a longitudinal mode, thus corresponding to a ripple pattern with crests perpendicular to the flow direction. In the first model, the destabilizing effect of fluid inertia is counterbalanced by the stabilizing mechanism due to gravity, whereas in the second one, the effect of grain inertia on sand transport is found to be the pertinent stabilizing process for sufficiently large particle Reynolds number. In addition, we find that a range of oblique modes is unstable and can couple to longitudinal modes in the nonlinear regime. A weakly nonlinear analysis indeed shows that the coupling between two oblique modes and a longitudinal one gives birth to complex three-dimensional sand ripples, which migrate in the flow direction at constant speed, preserving their shape. As a direct consequence, the three-dimensionality of the phenomenon cannot be neglected.

Citation: Langlois, V., and A. Valance (2005), Three-dimensionality of sand ripples under steady laminar shear flow, J. Geophys. Res., 110, F04S09, doi:10.1029/2004JF000278.

\section{Introduction}

[2] When subject to a fluid flow, erodible beds, that are initially flat, often deform and give rise to regular patterns resembling waves. Such bed forms can be observed in different natural contexts, like in tidal seas or in rivers. The understanding of the dynamics and morphology of these bed forms has been the subject of intense research activities, however a fuller description of the complex links between the fluid flow, the bed morphology and the sediment transport is still needed to be able to predict the long-term behavior of such patterns.

[3] Here, we address the issue of the influence of the three-dimensionality on the dynamics and morphology of sand bed forms. Most of the studies concerned only twodimensional bed forms, that is, structures that are invariant in the direction transverse to the flow. Yet, natural sand bed forms appearing under unidirectional flows often exhibit cross-stream variations and adopt complex three-dimensional shapes, such as dunes with sinuous crests [Wilbers and Brinke, 2003], crescent-shaped barchan dunes [Carling et al., 2000], and alternate sandbars [Nelson, 1990]. The evolution of transverse ripples to sinuous or "linguoid" patterns have also been experimentally observed by Raudkivi [1997].

Copyright 2005 by the American Geophysical Union. 0148-0227/05/2004JF000278
Recently Maddux et al. [2003a, 2003b] studied experimentally the flow over three-dimensional dunes and showed that the nature of the flow is greatly influenced by the dune shape. It seems therefore pertinent to investigate the physical mechanisms responsible for the evolution of a flat bed form toward these different types of three-dimensional (3-D) patterns. In this article, we focus on a simple configuration: a granular sand bed is sheared by a steady and laminar shear flow and we restrict our analysis to the small-scale bed forms, that is, the ripples. This configuration is, of course, far from natural situations where the flow is usually turbulent, however one expects to gain in the physical understanding of the destabilization mechanisms of a 3-D bed form. Indeed it can be considered as a canonical situation, and as it will be discussed in the conclusion, some of the "laminar" results can be extrapolated to the case of a turbulent flow. Besides, it is worth noting that ripples can be obtained under a laminar flow in laboratory experiments [Kuru et al., 1995].

[4] Before going further, let us recall the main theoretical works related to the formation of current-related ripples. Most studies focused on two-dimensional (2-D) configurations. The first attempts to understand the origin of sand ripple instability were based on potential flow models. It was shown [McLean, 1990] that the instability only occurs if an artificial lag between the flow and the bed profile is introduced. Further studies considered explicitly the turbu- 
lence of the flow [Richards, 1980]. These turbulent flow models are able to predict the ripple instability but the predicted wavelength of the sand pattern depends greatly on the way in which the turbulence is parameterized. Moreover, Charru et al. [2004] recently showed theoretically that the turbulence is not necessary for ripple instability. In the oscillatory case (that is, sea wave-related ripples), the linear stability analysis was performed by Blondeaux [1990] and the three-dimensionality of these ripples has been theoretically investigated by Vittori and Blondeaux [1992] and Roos and Blondeaux [2001] in the case of bidirectional or elliptical oscillatory flows. In particular, they studied the linear and nonlinear evolution of 3-D ripples. However, to our knowledge no similar three-dimensional theoretical analysis has been performed for current-related ripples.

[5] We present here such a theoretical study of the formation of 3-D sand ripples under a steady flow. The aim is to determine if the three-dimensionality of the system plays a role even in the case of a steady, unidirectional flow.

[6] The fluid flow above the bed forms is calculated analytically in 3-D. We propose two models for the description of the sediment transport. The first one is inspired from those developed in two dimensions [Fredsoe and Deigaard, 1992] and is based on a semiempirical law depending both on the local bed shear stress (which is calculated from the resolution of the hydrodynamic equations) and on the local bed slope. Within this model, we show that the linear stability analysis fails to predict realistic behaviors concerning the ripple wavelength. Therefore we introduce a second model which takes into account an additional mechanism depending on grain inertia and we compare the results with available experimental data. Finally, we investigate the nonlinear dynamics of the sand bed by performing a weakly nonlinear analysis.

[7] The paper is organized as follows. Section 2 presents the equations for the flow and the first model used for the sediment transport. Section 3 is devoted to the presentation of the linear stability analysis (within this basic model) and its prediction concerning the growth rate of the unstable modes. In section 4, we expose the second model for the sediment transport including grain inertia and report on the consequent outcomes concerning the linear stability of the sand bed. Section 5 deals with the nonlinear dynamics of the sand surface. Finally, the results are discussed in section 6.

\section{Presentation of the Basic Model}

[8] We introduce the axes $x, y$ and $z$ : $x$ corresponds to the direction of the flow, $y$ is the horizontal axis transverse to the flow and $z$ is the vertical axis. We consider a Couette configuration, that is, a laminar shear flow of thickness $L$ over a perturbed bed (this configuration can be considered as an approximation of a laminar boundary layer). The sketch of the studied system is presented in Figure 1. The flow obeys the hydrodynamic equations

$$
\left\{\begin{array}{l}
\rho \frac{\partial \mathbf{u}}{\partial t}+\rho(\mathbf{u} \cdot \boldsymbol{\nabla}) \mathbf{u}=-\boldsymbol{\nabla} p+\eta \boldsymbol{\nabla}^{2} \mathbf{u} \\
\boldsymbol{\nabla} \cdot \mathbf{u}=0,
\end{array}\right.
$$

$\mathbf{u}=(u, v, w)$ being the fluid velocity, $p$ the pressure, $\rho$ the volumetric mass of the fluid, and $\eta$ its dynamic viscosity
( $\nu=\eta / \rho$ being the kinematic viscosity). These equations are complemented with the following boundary conditions:

$$
\begin{cases}\mathbf{u}=\mathbf{0} & \text { at the bed surface } z=h(x, y, t) \\ \mathbf{u}=\gamma L \mathbf{e}_{\mathbf{x}} & \text { at the height } z=L,\end{cases}
$$

where $\mathbf{e}_{\mathbf{x}}$ is the unit vector defining the $x$ axis and $\gamma$ is the shear rate imposed by the flow.

[9] The sediment transport is a complex process which depends crucially on grain-grain and grain-fluid interactions. To date, there is no sound theoretical description of this process and the evaluation of the transport rate is based on semiempirical laws. In theoretical or numerical works, the horizontal flux of grains is usually assumed to be given by a power law of the Shields number [Fredsoe and Deigaard, 1992]:

$$
q=q_{b}\left[\Theta-\Theta_{c 0}\left(1+\frac{\partial_{x} h}{\mu_{s}}\right)\right]^{m} \times \mathcal{H}\left[\Theta-\Theta_{c 0}\left(1+\frac{\partial_{x} h}{\mu_{s}}\right)\right],
$$

where $\mathcal{H}$ is the Heaviside function, $\Theta$ is the dimensionless shear stress (or Shields parameter) at the sand surface, $\Theta_{c 0}$ is the critical Shields number corresponding to the onset of grain motion on a horizontal flat bed, and $\mu_{s}$ is the internal friction coefficient of the granular material. $q_{b}=$ $c \sqrt{(s-1) g d^{3}}$ is the reference grain flux, $c$ being a numerical constant, $s=\rho_{g} / \rho$ the relative density of the grains compared to that of the fluid, $g$ the gravitational acceleration and $d$ the diameter of the grains.

[10] The value of the exponent $m$ varies according to the authors. In the Meyer-Peter law, $m=3 / 2$ [Fredsoe and Deigaard, 1992], whereas Bagnold [1956] proposed a sediment transport law with $m=3$. The above law is only applicable in the case of a two-dimensional configuration (i.e., when the system is invariant in the direction $y$ perpendicular to the flow).

[11] Our purpose is to extend this transport law in the case of a three-dimensional configuration, where the system, and consequently the sand bed surface, is no longer invariant in the direction perpendicular to the flow. Our calculation is inspired from those of Fredsoe and Deigaard [1992] on the sediment transport on a transverse slope and of Kovacs and Parker [1994] who extended the Ashida-Michiue law for a 3 -D sediment flow rate in a turbulent flow.

[12] We first introduce a dimensionless bed shear stress vector defined by

$$
\Theta=\frac{\tau_{b}}{\rho g(s-1) d},
$$

with $\tau_{b}$ the bed shear stress (i.e., the flow shear stress calculated at the sand surface). The modulus of this dimensionless vector is referred to as the Shields parameter. Let us now enumerate the three forces acting on a single grain resting on the bed (see Figure 2). The first one is the immersed weight (i.e., the weight minus the buoyancy force):

$$
\mathbf{W}=-(s-1) \rho g d^{3} \mathbf{e}_{\mathbf{z}} .
$$

We define $\mathbf{n}$ as the local unit vector normal to the sand surface. The weight can be decomposed into a normal 


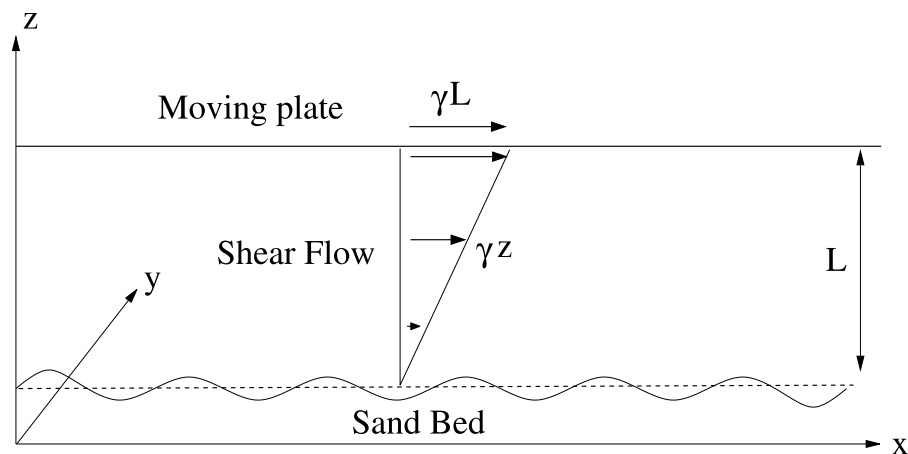

Figure 1. Sketch of the studied system.

component, $(\mathbf{W} \cdot \mathbf{n}) \mathbf{n}$, and a tangential one, $\mathbf{W}-(\mathbf{W} \cdot \mathbf{n}) \mathbf{n}$, which tends to destabilize the grain.

[13] The second force is the drag force exerted by the fluid, which is proportional to the bed shear stress:

$$
\mathbf{F}_{\mathbf{d}}=\xi(s-1) \rho g d^{3} \boldsymbol{\Theta} .
$$

The dimensionless constant $\xi$ is introduced to account for the local geometrical configuration of the bed around the grain (its value will be specified below). Finally, the third force is the frictional force experienced by the grain. Using the Coulomb friction law, at the onset of grain motion, one gets

$$
F_{f}=\mu_{s}(\mathbf{W} \cdot \mathbf{n}) \mathbf{u}_{\mathbf{f}}
$$

where $\mathbf{u}_{\mathbf{f}}$ is the unit vector corresponding to the grain motion direction and is given by

$$
\mathbf{u}_{\mathbf{f}}=\frac{\mathbf{F}_{\mathbf{d}}+\mathbf{W}-(\mathbf{W} \cdot \mathbf{n}) \mathbf{n}}{\left\|\mathbf{F}_{\mathbf{d}}+\mathbf{W}-(\mathbf{W} \cdot \mathbf{n}) \mathbf{n}\right\|} .
$$

[14] At the onset of grain motion, all forces should balance exactly, leading to

$$
\mu_{s}(\mathbf{W} \cdot \mathbf{n})=\left\|\mathbf{F}_{\mathbf{d}}+\mathbf{W}-(\mathbf{W} \cdot \mathbf{n}) \mathbf{n}\right\|
$$

At the dominant order, the above equation can be rewritten as

$$
\left\|\xi / \mu_{s} \Theta-\nabla h / \mu_{s}\right\|=1 .
$$

In the particular case of a flat horizontal bed, equation (10) leads to $\xi=\mu_{s} / \Theta_{c 0}$ (we remind the reader that $\Theta_{c 0}$ is the critical Shields number corresponding to the onset of grain motion on a flat horizontal bed). By analogy with the 2-D transport law, we shall assume the modulus of the grain flux to be a power law of the dimensionless quantity measuring the distance from the onset of grain motion, that is,

$$
\|\mathbf{q}\|=q_{b}\left[\left\|\boldsymbol{\Theta}-\frac{\Theta_{c 0}}{\mu_{s}} \boldsymbol{\nabla} h\right\|-\Theta_{c 0}\right]^{m} .
$$

Finally, we have to estimate the horizontal direction of the flux of grains. It is given by the horizontal projection of the sum of the destabilizing forces (weight and drag force), which yields at first order in $h: \xi \Theta_{\mathbf{h}}-\boldsymbol{\nabla} h$ (where $\boldsymbol{\Theta}_{\mathbf{h}}$ is the horizontal projection of the dimensionless shear stress). The horizontal flux of sand grains is therefore given by

$$
\mathbf{q}=q_{b}\left[\left\|\boldsymbol{\Theta}-\frac{\Theta_{c_{0}}}{\mu_{s}} \boldsymbol{\nabla} h\right\|-\Theta_{c_{0}}\right]^{m} \frac{\Theta_{h}-\frac{\Theta_{c_{0}}}{\mu_{s}} \boldsymbol{\nabla} h}{\left\|\Theta_{h}-\frac{\Theta_{c_{0}}}{\mu_{s}} \boldsymbol{\nabla} h\right\|} .
$$

For the further developments, the internal angle of stability will be taken equal to $30^{\circ}$ (or equivalently $\mu_{s}=0.58$ ) and the exponent $m$ equal to $3 / 2$.

[15] The closure of the model is given by the conservation of mass of grains, assuming that the average packing fraction of the bed $C_{0}$ is constant:

$$
C_{0} \frac{\partial h}{\partial t}=-\boldsymbol{\nabla} \cdot \mathbf{q}
$$

\section{Linear Stability Analysis}

[16] In this section, we study the linear stability of the basic state corresponding to the case of a flat horizontal bed. This basic state is characterized by a linear velocity profile $\mathbf{u}=\left(u_{0}, v_{0}, w_{0}\right)=\gamma z \mathbf{e}_{\mathbf{x}}$ and a transport rate given by

$$
\mathbf{q}_{0}=c \sqrt{(s-1) g d^{3}}\left(\Theta_{0}-\Theta_{c_{0}}\right)^{m} \mathbf{e}_{\mathbf{x}} .
$$

$\Theta_{0}=\nu \gamma /(g(s-1) d)$ is the dimensionless bed shear stress in the case of a flat bed. The pressure profile is hydrostatic:

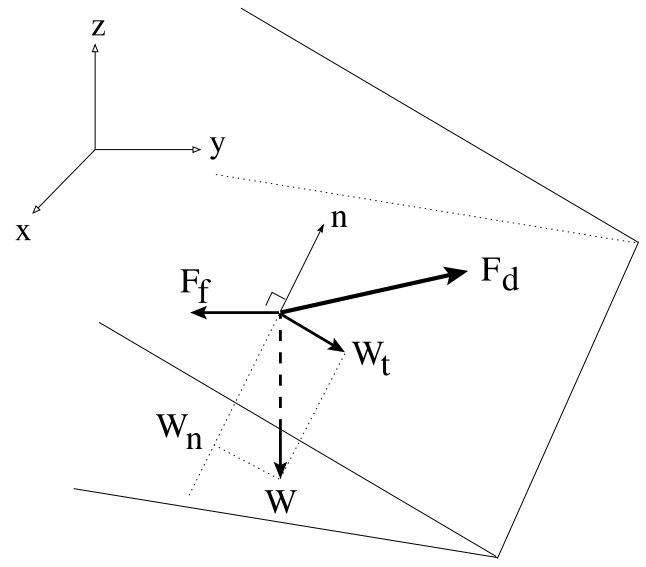

Figure 2. Sketch of forces acting on a grain. 
a

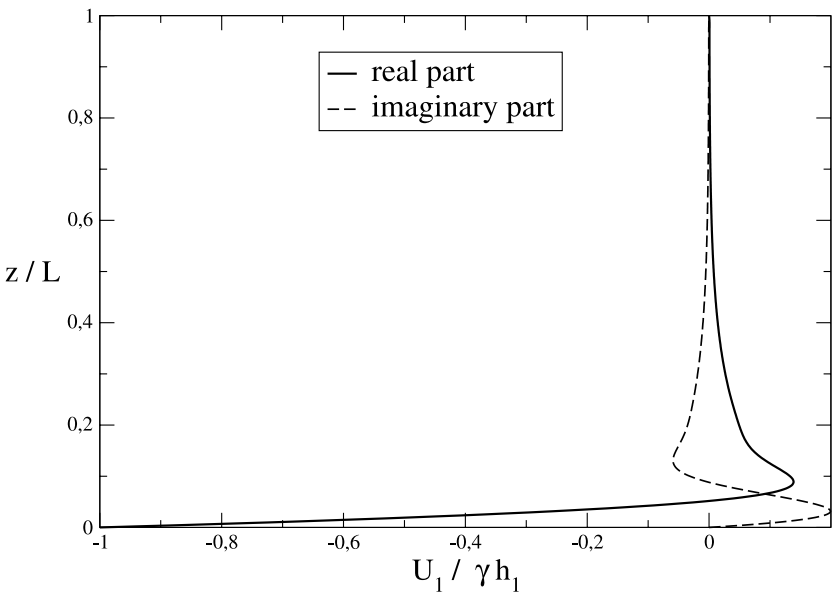

b

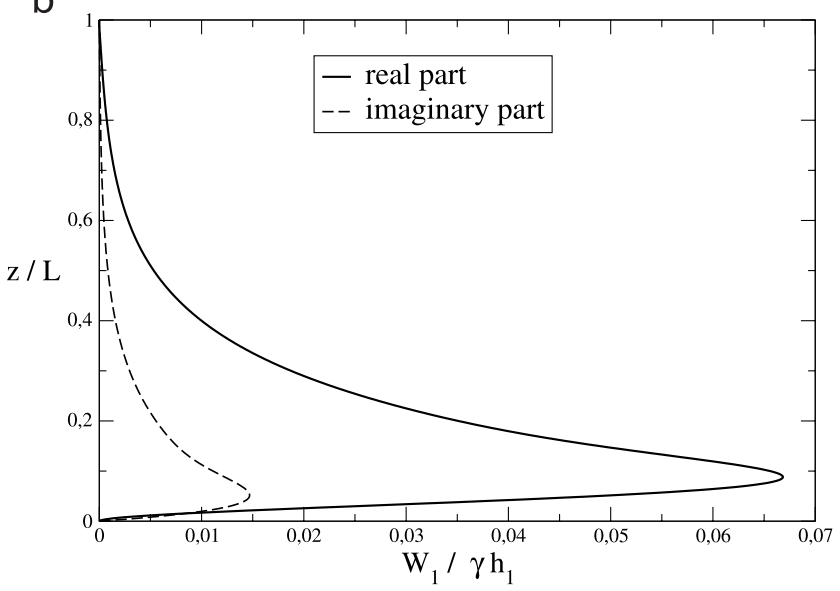

Figure 3. Perturbation velocity profiles for $k_{x} l_{\nu}=0.1$ and $k_{y}=0$ : (a) $x$ component and (b) $z$ component.

$p_{0}(z)=-\rho g z+$ cst (the constant being an arbitrary reference pressure).

[17] To investigate the stability of the planar sand bed, we introduce a small perturbation of the sand bed, namely,

$$
h(x, y, t)=h_{1} e^{i\left(k_{x} x+k_{y} y\right)+\omega t}+\text { c.c. },
$$

where $h_{1}$ is a small quantity, $\mathbf{k}=k_{x} \mathbf{e}_{\mathbf{x}}+k_{y} \mathbf{e}_{\mathbf{y}}$ is the wave vector of the perturbation, and $\omega$ its complex growth rate. The aim of the linear stability analysis is to obtain the dispersion relation, that is, the growth rate as a function of the wave vector. It is first necessary to calculate the fluid flow above the perturbed sand bed.

\subsection{Solution of the Hydrodynamic Equations Over a Perturbed Sand Bed}

[18] We introduce the following notations for the perturbed flow quantities:

$$
\begin{aligned}
(u, v, w, p)= & \left(u_{0}, 0,0, p_{0}\right) \\
& +\left[\left(U_{1}, V_{1}, W_{1}, P_{1}\right) e^{i\left(k_{x} x+k_{y} y\right)+\omega t}+\text { c.c. }\right] .
\end{aligned}
$$

We assume the quasi-stationarity of the hydrodynamic flow: the timescale of the movement of the bed is much larger than the timescale of the fluid motion. It means that the flow adapts instantaneously to any change of the bed profile, which allows one to calculate the flow as if the bed were fixed. Keeping only the linear terms in equation (1), we get the following coupled equations for $U_{1}, V_{1}, W_{1}$ and $P_{1}$ :

$$
\begin{gathered}
i k_{x} \gamma z U_{1}+\gamma W_{1}+i k_{x} \frac{P_{1}}{\rho}=\nu\left(\partial_{z}^{2}-k_{x}^{2}-k_{y}^{2}\right) U_{1} \\
i k_{x} \gamma z V_{1}+i k_{y} \frac{P_{1}}{\rho}=\nu\left(\partial_{z}^{2}-k_{x}^{2}-k_{y}^{2}\right) V_{1} \\
i k_{x} \gamma z W_{1}+\frac{1}{\rho} \partial_{z} P_{1}=\nu\left(\partial_{z}^{2}-k_{x}^{2}-k_{y}^{2}\right) W_{1} \\
i k_{x} U_{1}+i k_{y} V_{1}+\partial_{z} W_{1}=0 .
\end{gathered}
$$

In the linear approximation, the boundary conditions become

$$
\begin{array}{ll}
z=0: U_{1}=-\gamma h_{1}, & V_{1}=0, W_{1}=0 \\
z=L: U_{1}=0, & V_{1}=0, W_{1}=0 .
\end{array}
$$

[19] By combining equations (17)-(20), one can obtain a closed equation for $W_{1}$ :

$$
\left[\nu\left(\partial_{z}^{2}-k^{2}\right)-i k_{x} \gamma z\right]\left(\partial_{z}^{2} W_{1}-k^{2} W_{1}\right)=0,
$$

the solution of which can be expressed in terms of the Airy functions $A i$ and Bi [Abramovitz and Stegun, 1965]:

$$
\begin{aligned}
W_{1}(z)= & a_{1} e^{k z}+b_{1} e^{-k z}+\frac{e^{k z}}{2 k} \int_{0}^{z} \mathrm{~d} \zeta e^{-k \zeta}\left(a_{2} A i\left(\zeta^{\prime}\right)+b_{2} B i\left(\zeta^{\prime}\right)\right) \\
& -\frac{e^{-k z}}{2 k} \int_{0}^{z} \mathrm{~d} \zeta e^{k \zeta}\left(a_{2} A i\left(\zeta^{\prime}\right)+b_{2} B i\left(\zeta^{\prime}\right)\right),
\end{aligned}
$$

where $\zeta^{\prime}=e^{i \pi / 6}\left(k_{x} l_{v}\right)^{-2 / 3}\left(k_{x} \zeta-i k^{2} l_{v}^{2}\right), l_{v}=\sqrt{\nu / \gamma}$ is a "viscous" length and $k=\sqrt{k_{x}^{2}+k_{y}^{2}}$ is the wave number of the perturbation. $a_{1}, b_{1}, a_{2}$ and $b_{2}$ are integration constants to be determined using the boundary conditions. $U_{1}$ and $V_{1}$ can be also derived explicitly: the detailed calculation and analytical expressions are given in Appendix A. Figure 3 plots the profiles $U_{1}(z)$ and $W_{1}(z)$ in the two-dimensional case $\left(k_{y}=0\right)$ and for a small wave number $\left(k_{x} l_{\nu}=0.1\right)$. Note that this 3-D flow calculation was presented for the first time by Langlois and Valance [2005].

\subsection{Dispersion Relation}

[20] Let us first remind the reader that in the 2-D configuration [Charru et al., 2004], the instability arises from the phase lag between the shear stress and the bed profile. Indeed, as a result of mass conservation, a bump grows if the sand transport rate is greater on the stoss side than on the lee side. A sinusoidal perturbation is therefore expected to be amplified if the bed shear stress (which is in phase with the sand transport) is shifted upstream with respect to the bed profile.

[21] In the 2-D case, such a phase lag exists irrespective of the value of the wavelength: therefore, without the 


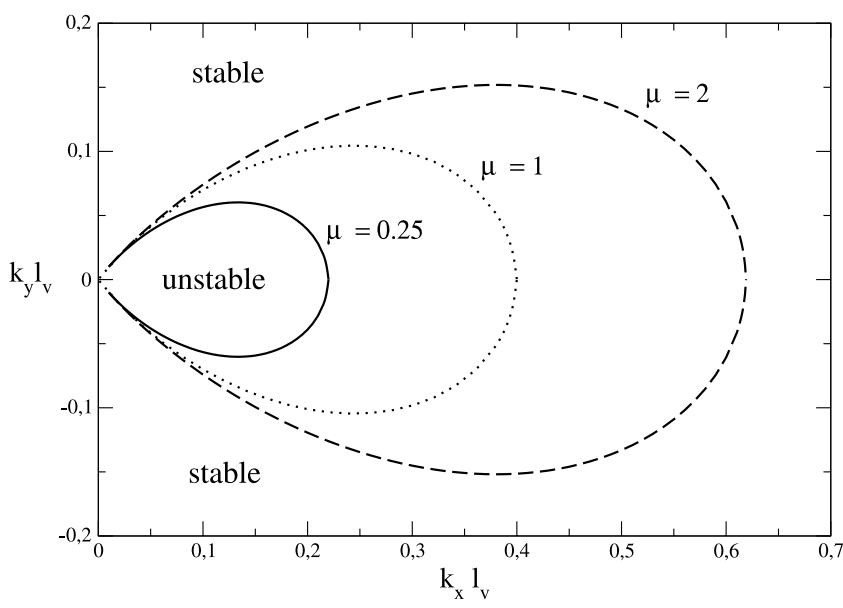

Figure 4. Marginal stability curves for different values of $\mu . \Theta_{c_{0}}=0.2$ and $\phi_{s}=30^{\circ}$.

presence of a stabilizing mechanism (such as gravity), all perturbations grow. Our purpose is to analyze what happens in the case of a three-dimensional flow.

[22] Once the velocity profile above the perturbed sand bed has been determined, it is possible to calculate the perturbed bed shear stress $\tau_{b_{1}}$ (we recall that $\tau_{b}=$ $\tau_{b_{0}}+\left[\tau_{b_{1}} e^{i\left(k_{x} x+k_{y} y\right)+\omega t}+\right.$ c.c. $\left.]\right)$. In order to simplify the further calculations, we shall focus on the particular situation where the height of the flow $L$ is much greater than the dimensions of the bed perturbation. Within this approximation, one can obtain analytical expressions of the shear stress in the longwavelength limit, that is, for $k l_{v} \ll 1$. One finds to the dominant order:

$$
\begin{gathered}
\frac{\tau_{b_{1 x}}}{h_{1} \tau_{b_{0}}}=l_{\nu}^{-2 / 3} k_{x}^{1 / 3} \frac{0.535 k_{x}^{2}-0.365 k_{y}^{2}}{k_{x}^{2}+k_{y}^{2}}(\sqrt{3}+i)+O\left(k l_{\nu}\right) \\
\frac{\tau_{b_{1 y}}}{h_{1} \tau_{b_{0}}}=-0.17 l_{\nu}^{-2 / 3} \frac{k_{x}^{4 / 3} k_{y}}{k_{x}^{2}+k_{y}^{2}}(\sqrt{3}+i)+O\left(k l_{\nu}\right),
\end{gathered}
$$

where $\tau_{b_{0}}=\eta \gamma$. Higher-order contributions are given in Appendix B. The aforementioned phase lag along $x$ (resp. $y$ ) between the bed shear stress and the bed profile corresponds to the imaginary part of $\tau_{b_{1,}}$ (resp. $\tau_{b_{1}}$ ): since it is non zero for each component, both of them are out of phase with the bed profile.

[23] Linearizing the transport law (equation (12)), and using mass conservation (equation (13)), we obtain the real and imaginary parts of the complex growth rate of the bed perturbation:

$$
\begin{aligned}
\frac{C_{0}}{q_{b}} \Re(\omega)= & m \mu^{m-1} \Theta_{c_{0}}^{m}\left[(1+\mu) k_{x} \Im\left(\frac{\tau_{b_{1} x}}{h_{1} \tau_{b_{0}}}\right)-\frac{k_{x}^{2}}{\mu_{s}}\right] \\
& +\mu^{m} \Theta_{c_{0}}^{m}\left[k_{y} \Im\left(\frac{\tau_{b_{1} y}}{h_{1} \tau_{b_{0}}}\right)-\Theta_{c_{0}}(1+\mu) \frac{k_{y}^{2}}{\mu_{s}}\right] \\
\frac{C_{0}}{q_{b}} \Im(\omega)= & -m \mu^{m-1} \Theta_{c_{0}}^{m}(1+\mu) k_{x} \Re\left(\frac{\tau_{b_{1} x}}{h_{1} \tau_{b_{0}}}\right) \\
& -\mu^{m} \Theta_{c_{0}}^{m} k_{y} \Re\left(\frac{\tau_{b_{1} y}}{h_{1} \tau_{b_{0}}}\right) .
\end{aligned}
$$

[24] We have introduced the parameter $\mu=\left(\Theta_{0}-\Theta_{c_{0}}\right) /$ $\Theta_{c_{0}}$ which measures the relative distance from the threshold of grain motion, and will be referred to as the relative shear stress excess.

[25] The complex parameter $\omega$ contains two pieces of information: the phase velocity of the perturbation given by the imaginary part (i.e., by $\Im(\omega)$ ) and its growth rate given by the real part (i.e., by $\Re(\omega)$ ). A negative growth rate indicates that the bed is stable with respect to this perturbation mode (its amplitude decays exponentially) whereas an amplified mode corresponds to a positive growth rate.

\subsubsection{Growth Rate: Selection of a Wavelength}

[26] Equation (26) consists of two contributions involving the grain flux in the $x$ direction and the $y$ direction, respectively. Each of these contributions depends on the bed shear stress and on the slope of the bed. Let us first analyze the terms that depend on the slope: they are real, negative and proportional to the squared wave number (terms proportional to $k_{x}^{2} / \mu_{s}$ and $k_{y}^{2} / \mu_{s}$ in equation (26). Therefore the effect of the gravity is to damp the largest wave numbers, that is, the smallest wavelengths (corresponding to the highest slopes): it tends to smooth the sand surface. The other terms in equation (26) depend on $\tau_{b_{1}}$. Referring to expressions (24)-(25), one can note that for small $k, \tau_{b_{1 x}}$ plays a destabilizing role whereas $\tau_{b_{1 y}}$ stabilizes the sand bed. The competition between these two opposite mechanisms leads to the existence of a region of amplified modes in the parameter space $\left(k_{x}, k_{y}\right)$.

[27] This region can be seen on the stability diagram: Figure 4 plots the marginal stability curve for different values of $\mu$, that is, the curve where $\Re(\omega)=0$. The curve delimits an inner and outer region: the bed is unstable with respect to modes contained in the inner region while it is stable with respect to those of the outer region. The unstable region expands as the shear stress excess $\mu$ increases. One can also note that for small $k$, pure longitudinal modes (i.e., $k_{y}=0$ ) are always amplified whereas pure transverse modes (i.e., $k_{x}=0$ ) are damped. For larger $k$, the longitudinal modes are stabilized by the gravitational effect, as found in the 2-D analysis [see, e.g., Charru et al., 2004; Valance and Langlois, 2005].

[28] The marginal curves shown in Figure 4 were plotted for particular values of the friction coefficient $\mu_{s}$ (or equivalently the internal angle of friction $\phi_{s}=\arctan \left(\mu_{s}\right)$ ) and of the critical Shields number $\Theta_{c_{0}}$. We chose $\phi_{s}=30^{\circ}$ (corresponding to the maximal angle of stability of a pile) and $\Theta_{c 0}=0.2$. Note that the experimental determination of these parameters is rather controversial. For example, recent experimental observations tend to show that much greater values of $\phi_{s}$ should be used (up to $60^{\circ}$ ) [Loiseleux et al., 2004]. The spectrum of reported values for the critical Shields number is quite large (from 0.05 to 0.35 ) as well. It is therefore instructive to study the influence of these parameters on the marginal stability curve. The results are shown in Figures 5 and 6. It turns out that the value of the critical Shields number does not have much influence on the extension of the unstable region. On the contrary, the unstable region is very sensitive to a change of the internal angle of friction: it expands as $\phi_{s}$ increases. Therefore it appears crucial that careful experimental studies on the determination of the internal angle of friction should be conducted.

[29] Besides the extension of the unstable region, it is important to determine the fastest-growing mode, because it 


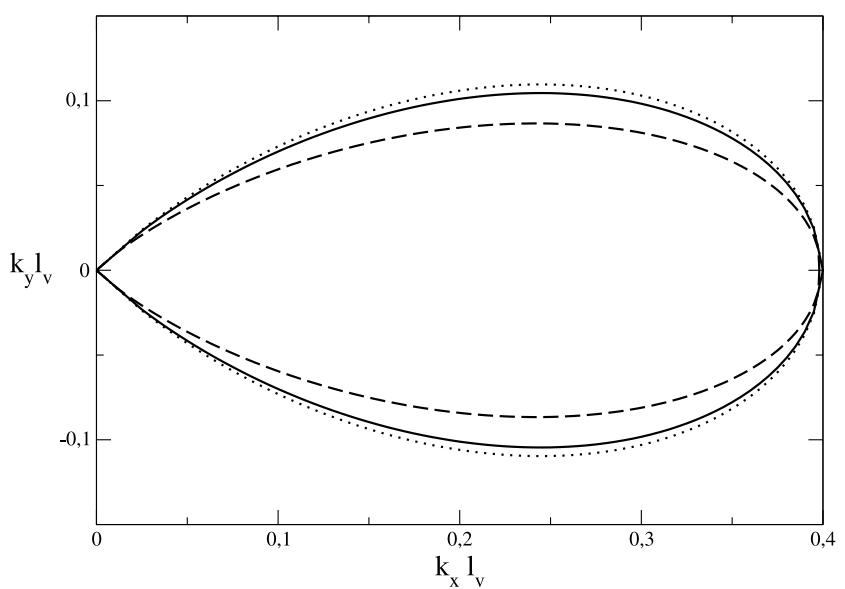

Figure 5. Marginal stability curves for different values of $\Theta_{c_{c}}$ : Dotted curve is $\Theta_{c_{0}}=0.05$, solid curve is $\Theta_{c_{0}}=0.2$, and dashed curve is $\Theta_{c_{0}}=1$. Here $\mu=1$ and $\phi_{s}=30^{\circ}$.

is expected to give the order of magnitude of the ripple wavelength in the first stages of the instability development. Figure 7 plots the real and imaginary parts of the growth rate versus $k_{x}$ for $k_{y}=0$ and versus $k_{y}$ for $k_{x}=k_{x}^{\max }$. Despite the presence of amplified oblique modes (i.e., $k_{y} \neq 0$ ), the fastest-growing mode is found to be longitudinal (i.e., $k_{y}=0$ ) and therefore corresponds to ripples having crests perpendicular to the flow. This is the same result as that found by Roos and Blondeaux [2001] with an oscillatory flow. This finding seems to be rather consistent with the experimental observations made for unidirectional flows in wide channels: the ripples appearing at the first stages of the instability have crests perpendicular to the flow, before evolving to more complex patterns [Kuru et al., 1995]. The wavelength of the fastest-growing mode can be easily calculated and one finds

$$
\lambda_{\max }=\frac{30 l_{v}}{\mu_{s}^{3 / 2}(1+\mu)^{3 / 2}}=\frac{1}{\sqrt{g(s-1) d}} \frac{30 \nu}{\Theta_{c_{0}}^{1 / 2} \mu_{s}^{3 / 2}(1+\mu)^{2}} .
$$

This result is the same as that found in the 2-D configuration analysis [Charru et al., 2004; Valance and Langlois, 2005]. Let us mention that the most amplified

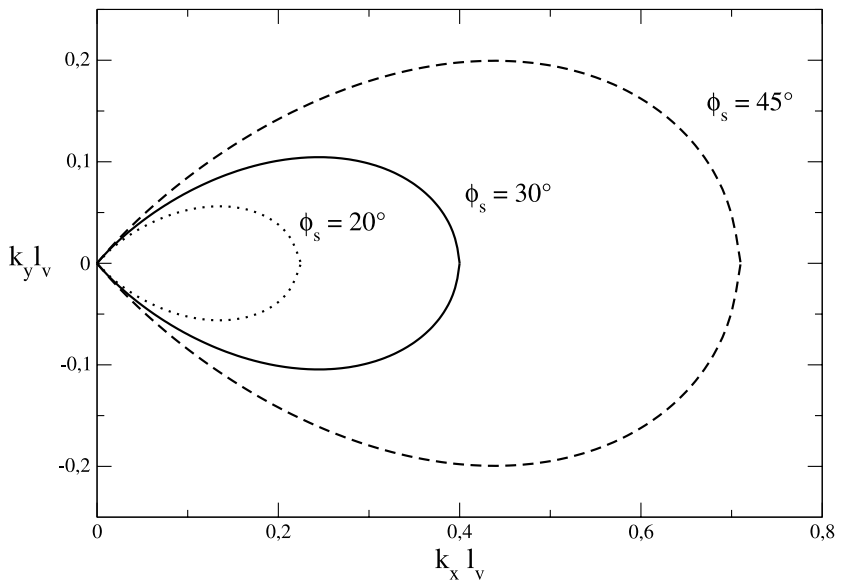

Figure 6. Marginal stability curves for different values of $\phi_{s}$. Here $\mu=1$ and $\Theta_{c_{0}}=0.2$. wavelength depends both on the grain and flow properties. Hence it appears that the selected wavelength decreases when the diameter of the grains or the shear stress excess increase. In Figure 8, we have plotted the evolution of the fastest-growing wavelength varying the grain diameter and the shear stress excess. In the case of a water flow (i.e., $v=$ $10^{-6} \mathrm{~m}^{2} / \mathrm{s}$ ), we obtain, for $\mu=1.1$ and $d=110 \mu \mathrm{m}, \lambda_{\max }=$ $0.9 \mathrm{~mm}$, which is a rather small value compared to experimental observations where the typical wavelength is of order of a few centimeters, at the initiation of the instability [Kuru et al., 1995; Loiseleux et al., 2004]. The latter paper gives an initial wavelength of $4 \mathrm{~cm}$ with $\mu=1.1$ and $d=110 \mu \mathrm{m}$. Moreover, the observations tend to show that the wavelength increases with the excess shear stress, contrary to the theoretical prediction. It is therefore necessary to improve the description of the sand transport. We propose in the next section an improvement of the sand transport modeling by taking into account the grain inertia.

\subsubsection{Migration Speed}

[30] The imaginary part of $\omega$ contains information about the migration of the ripples. In Figure 9 we plotted the sign of $\Im(\omega)$ for $\mu=1$. As can be seen in equation (15), the ripple

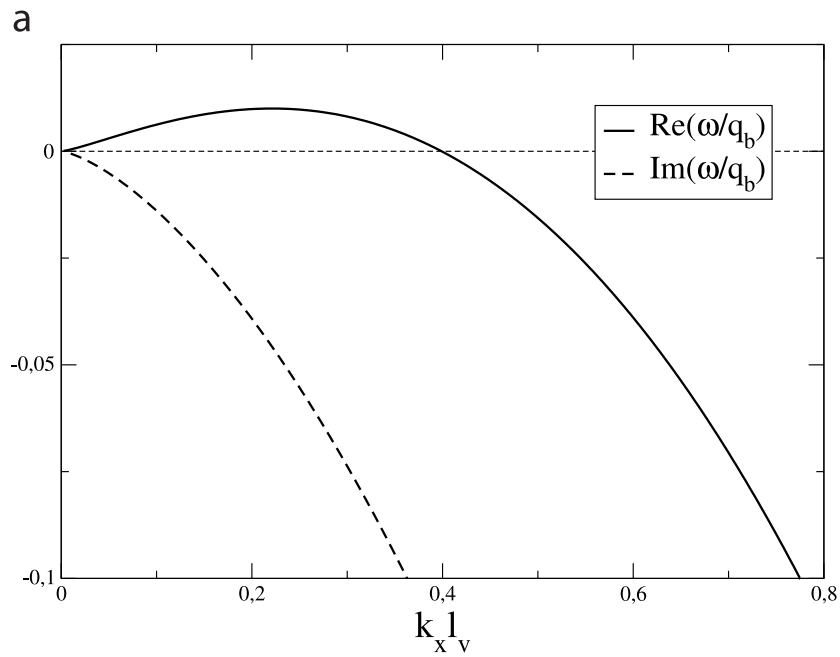

b

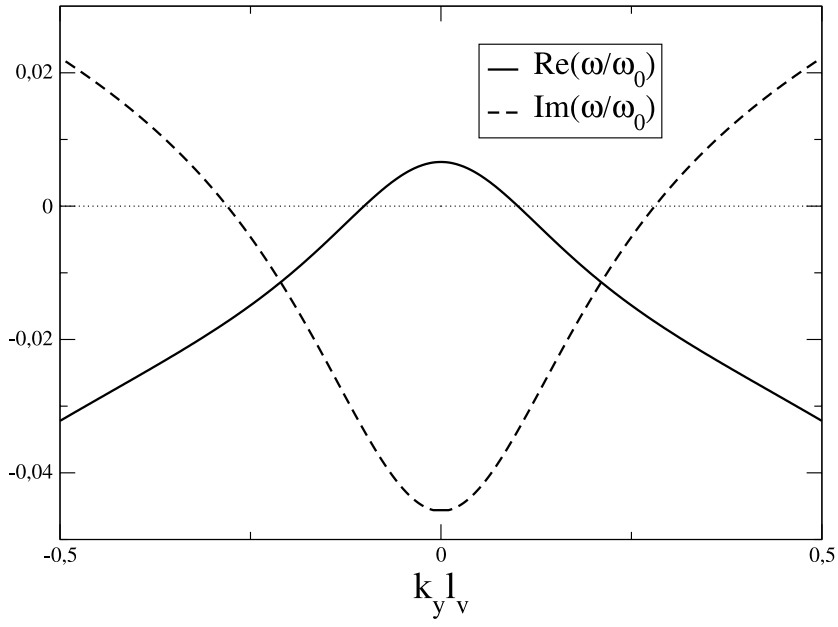

Figure 7. Growth rate for $\mu=1\left(\omega_{0}=q_{b} / l_{\nu}^{2}\right)$ versus (a) $k_{x}$ for $k_{y}=0$ and (b) $k_{y}$ for $k_{x}=k_{x}^{\max }$. 


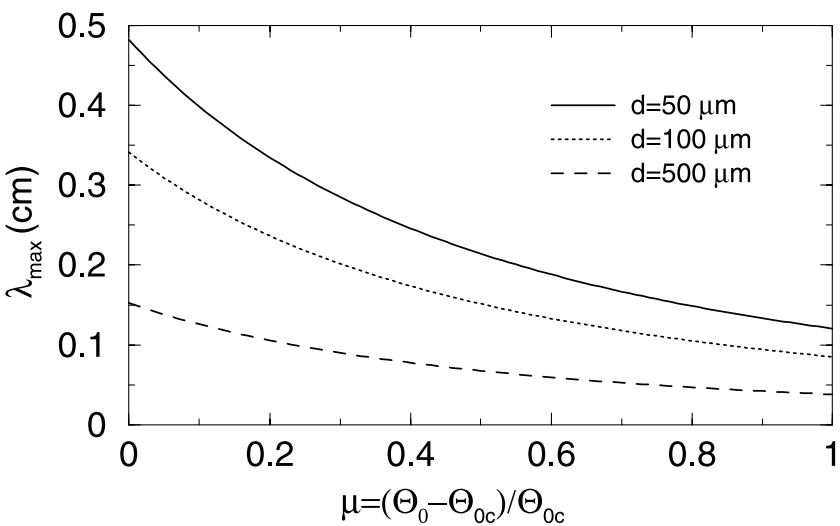

Figure 8. Evolution of the fastest-growing wavelength with the shear stress excess $\mu$ for different values of the grain diameter (in the gravitational regime).

migrates downstream if $\Im(\omega)<0$ and upstream if $\Im(\omega)>0$. One can note on the diagram that all amplified modes migrate downstream whereas ripples roughly parallel to the main flow migrate upstream.

[31] Moreover, the imaginary part of the growth rate is plotted on Figure 7 versus $k_{x}$ for $k_{y}=0$ and versus $k_{y}$ for $k_{x}=$ $k_{x}^{\max }$. In the case $k_{y}=0$; that is, for longitudinal modes, the migration speed reads to dominant order:

$$
V_{d}=-\frac{\Im(\omega)}{k_{x}}=0.93 \frac{q_{b}}{C_{0}} m \mu^{m-1}(1+\mu) l_{\nu}^{-2 / 3} \Theta_{c_{0}}^{m} k_{x}^{1 / 3}
$$

[32] This velocity grows with the wave number of the perturbation: the larger the ripples, the more slowly they migrate.

\section{Sand Transport Model Including Grain Inertia}

[33] The predictions of the above calculation seem to fail for typical experimental parameters. It turns out that the grain inertia, neglected in the former analysis, is not negligible. Indeed, the particle Reynolds number $\left(R e_{p}=\right.$

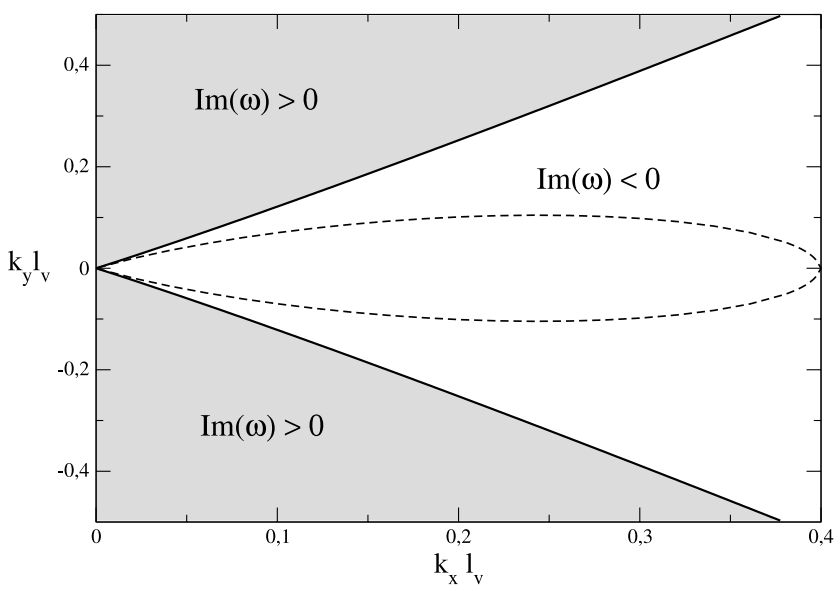

Figure 9. Sign of the imaginary part of the growth rate for $\mu=1$. Gray region is the upstream migration, and white region is the downstream migration. The dotted curve is the marginal stability curve. $\gamma d^{2} / \nu$ ) at the onset of grain motion becomes greater than unity when the grain diameter exceeds $50 \mu \mathrm{m}$. It is therefore likely that the grain inertia plays a role in the sediment transport process. In particular, one can expect that because of inertia, the grain flux does not adapt instantaneously to the local bed shear stress. We therefore propose a new model taking into account this process.

[34] As suggested above, in a nonequilibrium situation, the grain flux does not instantaneously reach its equilibrium value $\mathbf{q}^{e q}$. Indeed, if the fluid velocity increases, new grains from the bed are set into motion and it takes some time for them to reach the flow velocity. This characteristic equilibrium time can be associated with an equilibrium length $l_{e q}$ (which corresponds to the distance necessary for the grains, initially at rest, to equilibrate their velocity with that of the fluid). Furthermore, one expects that this relaxation process is of significative importance only in the main direction of the flow. As a consequence, we propose the following relaxation law for the grain flux:

$$
\begin{gathered}
\frac{\partial q_{x}}{\partial x}=-\frac{q_{x}-q_{x}^{e q}}{l_{e q}} \\
q_{y}=q_{y}^{e q}
\end{gathered}
$$

where $l_{e q}$ is the characteristic length needed for the grain flux in the flow direction, $q_{x}$, to reach its equilibrium value $q_{x}^{e q}$. The equilibrium transport rate $\mathbf{q}^{e q}$ is taken to be equal to that established in section 2. The estimation of the length $l_{e q}$ is quite complex because of the intricate interactions between the fluid and the grains. However, we can get a crude approximation assuming that the moving grains roll on the sand bed surface and that their equilibrium velocity is given, to zero order, by $\gamma d / 2$ [Valance and Langlois, 2005]. Within these approximations, we obtain

$$
l_{e q}=f\left(R e_{p}\right) s d
$$

where $f$ is a function of the particle Reynolds number $R e_{p}$ which is constant for high values of $R e_{p}$ and scales as $R e_{p}$ for lower ones [Valance and Langlois, 2005]. Figure 10 gives the value of $R e_{p}$ at the grain motion threshold as a function of the grain diameter, while the evolution of this equilibrium length with $R e_{p}$ is plotted in Figure 11. It is

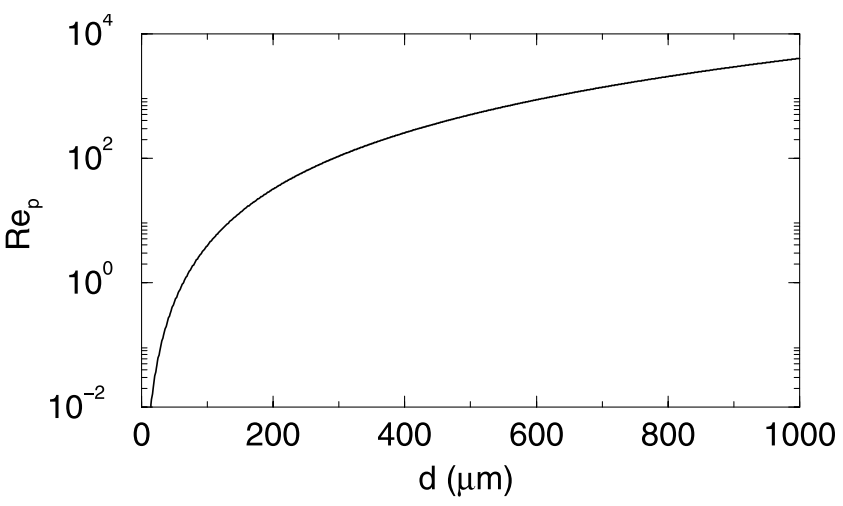

Figure 10. Particle Reynolds number calculated at the onset of grain motion as a function of $d$. 


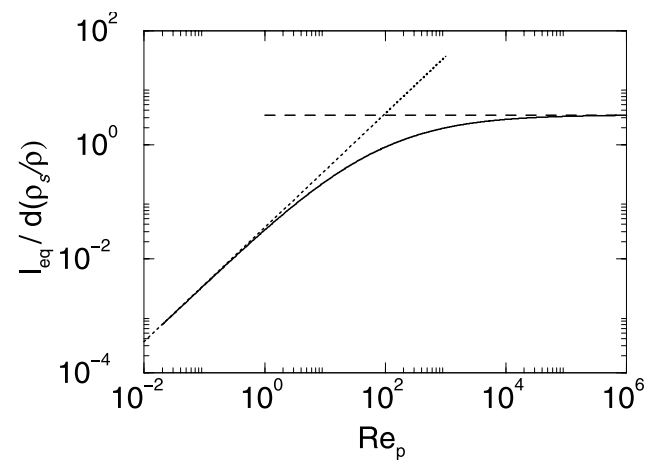

Figure 11. Equilibrium length as a function of the particle Reynolds number. For small values of $\operatorname{Re}_{p}\left(\operatorname{Re}_{p}<10\right), l_{\text {eq }} \sim$ $R e_{p} d$ (dotted curve), and for large values $\left(R e_{p}>10^{4}\right), l_{e q}$ tends to be independent of $R e_{p}$.

worth noting that this equilibrium length is analogous to the "saturation length" of the flux introduced in the context of aeolian sand transport [Sauermann et al., 2001; Andreotti et al., 2002].

[35] Within the framework of this new model for the sand transport, we can again perform a linear stability analysis of the planar sand bed. We now obtain for the dispersion relation the following result:

$$
\begin{aligned}
\frac{C_{0}}{q_{b}} \omega= & -i k_{x} m \mu^{m-1} \Theta_{c_{0}}^{m}\left((1+\mu) \frac{\tau_{b_{1} x}}{\tau_{b_{0}}}-\frac{i k_{x}}{\mu_{s}}\right)\left(\frac{1-i k_{x} l_{e q}}{1+k_{x}^{2} l_{e q}^{2}}\right) \\
& -i k_{y} \mu^{m} \Theta_{c_{0}}^{m}\left(\frac{\tau_{b_{1} y}}{\tau_{b_{0}}}-\Theta_{c_{0}}(1+\mu) \frac{i k_{y}}{\mu_{s}}\right) .
\end{aligned}
$$

[36] The grain inertia adds the factor $\left(\frac{1-i k_{x} l_{e q}}{1+k_{x}^{2} l_{e q}^{2}}\right)$ to the " $x$ component" of the growth rate previously obtained (equation (26)). This term introduces a new stabilizing mechanism, in addition to the gravitational one. This mechanism is expected to be relevant and to prevail over gravity for

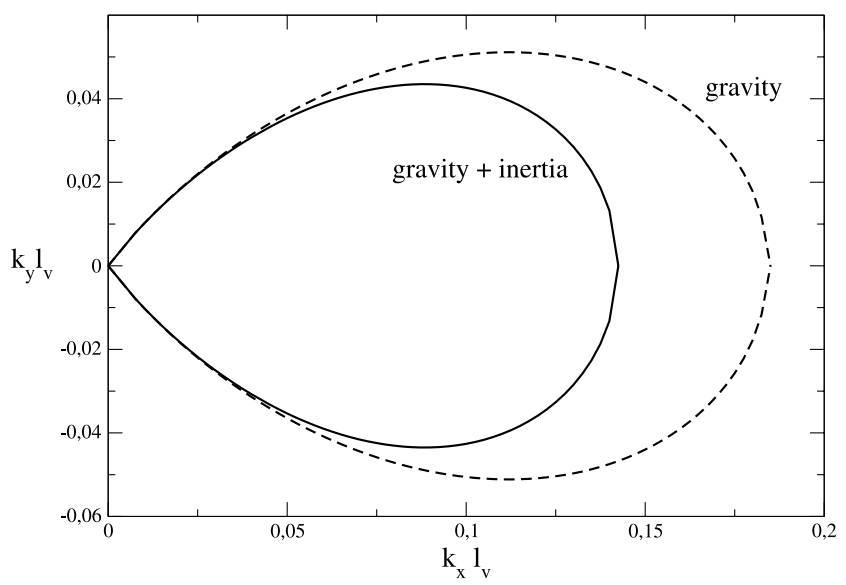

Figure 12. Marginal stability curves, taking into account the effects of gravity only (dotted curve) and of both gravity and grain inertia (solid curve). Here $s=2.7, d=50 \mu \mathrm{m}$, and $\mu=0.1\left(\operatorname{Re}_{p}=2.3\right)$.

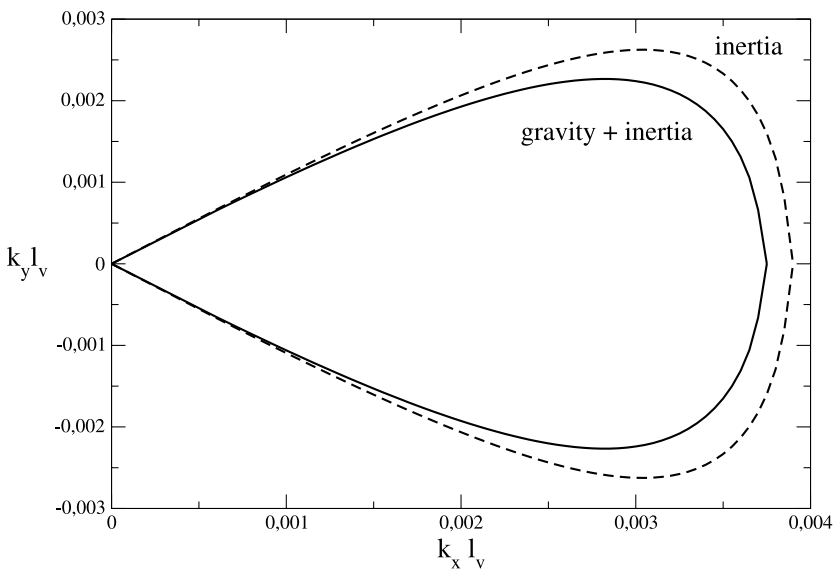

Figure 13. Marginal stability curves, taking into account the effects of inertia only (dotted curve) and of both gravity and grain inertia (solid curve). Here $s=2.7, d=200 \mu \mathrm{m}$, and $\mu=1\left(\operatorname{Re}_{p}=267\right)$.

large enough particle Reynolds number. Figures 12 and 13 compare the unstable regions obtained by taking into account separately gravity or grain inertia, and combining both effects. In the first case (Figure 12), the particle Reynolds number has been taken to be small. In this situation, adding the inertial effect does not affect the unstable region much: the gravity is the predominant stabilizing mechanism. In this gravity regime, the fastestgrowing mode is given by equation (28). In the second case (Figure 13), we chose parameters such that the particle Reynolds number is much larger. Here, adding the effect of gravity to the inertial one does not affect the shape of the unstable region. Grain inertia is therefore the pertinent stabilizing mechanism at large particle Reynolds number. In this regime, the fastest-growing mode is still a longitudinal mode but has a different length scale. It is given by

$$
\lambda_{\max } \simeq 19 l_{\text {eq }} .
$$

As can be seen in Figure 14, the wavelength of the fastestgrowing mode increases when the shear stress excess $\mu$ and the grain diameter increase, which is contrary to what we obtained in the gravity regime and more compatible with the experimental observations. In the case of a water flow (i.e., $v=10^{-6} \mathrm{~m}^{2} / \mathrm{s}$ ), for $d=200 \mu \mathrm{m}, s=2.7$, and $\mu=1$, the wavelength is found to be $\lambda_{\max }=1 \mathrm{~cm}$. This prediction gives a better agreement, although it is still quite small, with the typical experimental values. However, one cannot expect the prediction to be very accurate since it is based on a rough estimation of the equilibrium length.

\section{Weakly Nonlinear Analysis}

[37] An important result from the 3-D linear stability analysis is the existence of amplified oblique modes. Since they are not growing as fast as the longitudinal ones, they are not expected to play a role in the linear regime, but they could couple to longitudinal modes in the nonlinear regime. If such coupling exists, one may wonder what the long-term dynamics and the obtained patterns will be. 


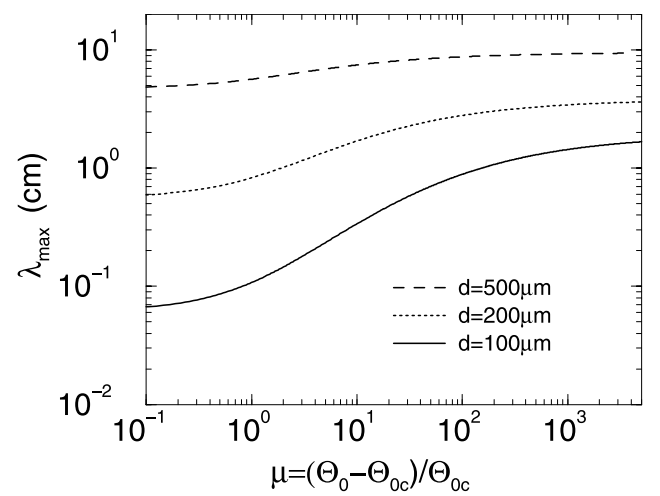

Figure 14. Evolution of the fastest-growing wavelength with the shear stress excess $\mu$ for different values of the grain diameter (in the inertial regime).

[38] We present here a weakly nonlinear analysis, already introduced by Langlois and Valance [2005]. We will restrict our analysis to a rather simple configuration, by focusing on the nonlinear interaction between three modes: one longitudinal mode and two oblique modes. The coupling between these modes is resonant if the sum of the latter two wave vectors equals the former. This is the very same configuration as that previously investigated under oscillatory flow by Vittori and Blondeaux [1992] and Roos and Blondeaux [2001]. Furthermore, we will choose two symmetrical oblique modes. We introduce the following notations for the wave vectors of three modes:

$$
\begin{gathered}
\mathbf{k}_{1}=k_{x} \mathbf{e}_{x} \\
\mathbf{k}_{2}=\frac{k_{x}}{2} \mathbf{e}_{x}+k_{y} \mathbf{e}_{y} \\
\mathbf{k}_{3}=\frac{k_{x}}{2} \mathbf{e}_{x}-k_{y} \mathbf{e}_{y} .
\end{gathered}
$$

Their complex amplitudes are respectively $A_{1}, A_{2}$, and $A_{3}$. [39] We thus consider the following structure for the sand bed surface perturbation:

$$
h_{1}(x, y, t)=\left(A_{1}(t) e^{i \mathbf{k}_{1} \cdot \mathbf{r}}+A_{2}(t) e^{i \mathbf{k}_{2} \cdot \mathbf{r}}+A_{3}(t) e^{i \mathbf{k}_{3} \cdot \mathbf{r}}\right)+\text { c.c. },
$$

with $\mathbf{r}=x \mathbf{e}_{\mathbf{x}}+y \mathbf{e}_{\mathbf{y}}$ designing the horizontal position. In order to investigate the temporal evolution of this perturbation, we perform a weakly nonlinear analysis. Following Vittori and Blondeaux [1992], we restrict our study to the nonlinearities induced by the transport law (12), neglecting those created by the fluid flow. Indeed, if we consider modes close enough to the marginal stability curve, the nonlinear hydrodynamical terms will appear at higher order. Hence the flow is calculated using the linear approximation while the sediment transport is estimated taking into account the nonlinearities. Furthermore, the grain inertia effect will be neglected in this nonlinear analysis. Finally, the long-wave limit $\left(k l_{\nu} \ll 1\right)$ and the infinite depth approximation are still adopted.
[40] Inserting equation (37) into equations (12) and (13), we get three coupled nonlinear equations for the amplitudes $A_{1}, A_{2}$ and $A_{3}$ :

$$
\begin{aligned}
& \frac{d A_{1}}{d t}=\omega_{1} A_{1}+K_{1} A_{2} A_{3} \\
& \frac{d A_{2}}{d t}=\omega_{2} A_{2}+K_{2} A_{1} A_{3}^{*} \\
& \frac{d A_{3}}{d t}=\omega_{3} A_{3}+K_{3} A_{1} A_{2}^{*},
\end{aligned}
$$

where $*$ denotes the complex conjugation. The coupling coefficients $K_{1}, K_{2}$ and $K_{3}$ can be calculated analytically as a function of the bed shear stress calculated at the first order (see detailed expressions in Appendix C). Because of the symmetry, $\tau_{2}=\tau_{3}$ and $\omega_{2}=\omega_{3}$ such that equations for $A_{2}$ and $A_{3}$ are identical. For simplicity, we restrict our analysis to the situation where $A_{2}=A_{3}$.

[41] Introducing the real-valued amplitude $a_{i}$ and the phase $\phi_{i}$ of the complex amplitude $A_{i}$ (i.e., $A_{i}=a_{i} e^{i \phi_{i}}$ ), we can rewrite equations (38)-(40) as

$$
\begin{gathered}
\frac{d a_{1}}{d t}=\Re\left(\omega_{1}\right) a_{1}+\kappa_{1}(\Delta \phi) a_{2}^{2}, \\
\frac{d a_{2}}{d t}=\Re\left(\omega_{2}\right) a_{2}+\kappa_{2}(\Delta \phi) a_{1} a_{2},
\end{gathered}
$$

$$
\begin{aligned}
\frac{d(\Delta \phi)}{d t}= & \Im\left(2 \omega_{2}-\omega_{1}\right)-\kappa_{3}\left(a_{1}, a_{2}\right) \sin (\Delta \phi) \\
& +\kappa_{4}\left(a_{1}, a_{2}\right) \cos (\Delta \phi),
\end{aligned}
$$

where $\Delta \phi=2 \phi_{2}-\phi_{1}$. The expressions of the coefficients $\kappa_{1}, \kappa_{2}, \kappa_{3}$ and $\kappa_{4}$ can be found in Appendix C. Let us analyze the dynamics of the bed surface described by such equations. When the amplitudes $a_{1}$ and $a_{2}$ are small enough, the nonlinear terms are not pertinent so that the two modes grow or decay, according to their growth rate $\Re\left(\omega_{i}\right)$. In the case of unstable modes, they will grow exponentially until the nonlinear terms become large enough and enter the dynamics. The nonlinearities can either enhance or saturate the exponential growth depending on the sign of the coupling coefficients $\kappa_{1}$ and $\kappa_{2}$, which depends in a complicated way on the wave number of both modes and on their relative phase $\Delta \phi$. If both modes are linearly unstable, as soon as $\kappa_{1}$ or $\kappa_{2}$ is positive, $a_{1}$ or $a_{2}$ will have an explosive growth (i.e., faster than an exponential one) and the nonlinear analysis will break down. However, if both $\kappa_{1}$ and $\kappa_{2}$ are negative, the nonlinear term can exactly balance the unstable linear term leading to steady threedimensional sand ripples.

[42] These steady patterns correspond to the stationary solutions of equations (41)-(43), that is,

$$
\frac{d a_{1}}{d t}=\frac{d a_{2}}{d t}=\frac{d(\Delta \phi)}{d t}=0 .
$$




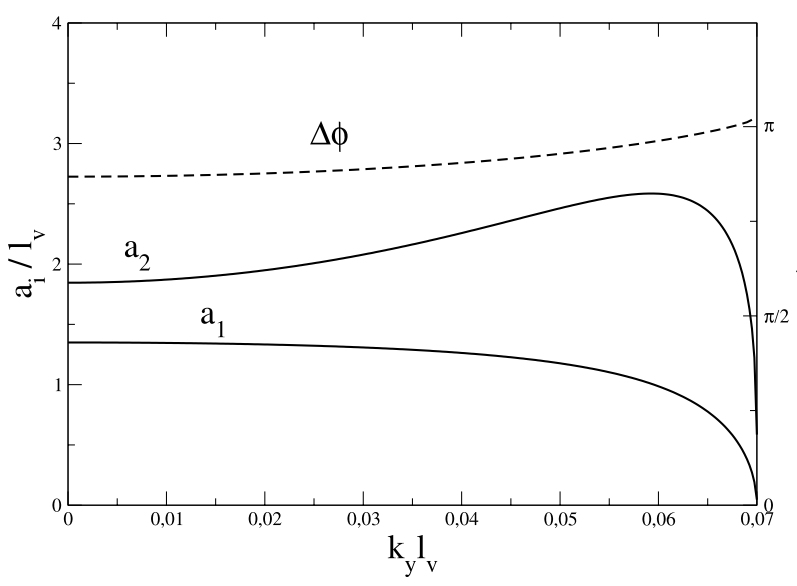

Figure 15. Amplitudes $a_{1}$ and $a_{2}$ and phase shift $\Delta \phi$ corresponding to steady state solutions as a function $k_{y}$ for a given value of $k_{x}$. Parameters are $k_{x} l_{v}=0.1$ and $\mu=1$.

In fact, these patterns migrate along the flow direction at a constant speed given by

$$
v_{d}=\frac{2}{k_{x}} \frac{d \phi_{2}}{d t}=\frac{1}{k_{x}} \frac{d \phi_{1}}{d t} .
$$

We have determined the domain of existence of these solutions in the parameter space $(\mu, \mathbf{k})$ and have found that there always exist such stationary solutions as soon as the modes $k_{1}$ and $k_{2}$ lie in the linearly unstable region (i.e., $\left.\Re\left(\omega_{i}\right)>0\right)$. We have presented, in Figure 15, the amplitude $a_{1}, a_{2}$ and the phase shift $\Delta \phi$ corresponding to the stationary solution, as a function of $k_{y}$ for given values of $k_{x}$ and $\mu$. One should point out that $k_{y}$ varies from 0 to the critical value $k_{y}^{c}$ defined by $\Re\left(\omega\left(k_{y}^{c}\right)\right)=0$. Above this critical value, the mode $k_{2}$ becomes linearly stable. The patterns corresponding to these steady solutions can exhibit a large variety of shapes. The pattern morphology depends crucially on the ratio $a_{1} / a_{2}$ and on the phase shift $\Delta \phi$. Figure 16 gives some examples of ripple patterns obtained for different typical values of $a_{1} / a_{2}$ and $\Delta \phi$. These patterns are similar to those obtained under an oscillatory flow by Vittori and Blondeaux [1992].

[43] It is worth noting that in the framework of our model, steady three-dimensional patterns that migrate in the flow direction (without growing or decaying) can exist. Although the analysis was restricted to the interaction of a triad of modes, this result suggests that 3-D patterns can arise from a flat bed in this hydrodynamic setting. It would be useful to conduct a full nonlinear analysis in order to determine whether such 3-D patterns can be stable.

\section{Conclusion}

[44] In this paper we presented a linear and nonlinear analysis of the problem of sand ripple formation in the case of a laminar shear flow. We treated the full three-dimensional configuration (3-D flow over 3-D sand pattern) and introduced a new description for the sediment transport taking into account the grain inertia. The linear stability analysis reveals that although the fastest-growing mode is longitudinal, a range of oblique modes can be amplified. Furthermore, we identify two distinct regimes where the most amplified wavelength exhibits different scalings: in the gravitational regime, the wavelength decreases for increasing grain diameter and increasing shear stress excess, whereas in the inertial regime, the wavelength grows with the grain diameter and the shear stress excess. When compared to available experimental data, the first model appears to fail to predict a reasonable behavior, whereas the second model shows a better agreement with experiments.
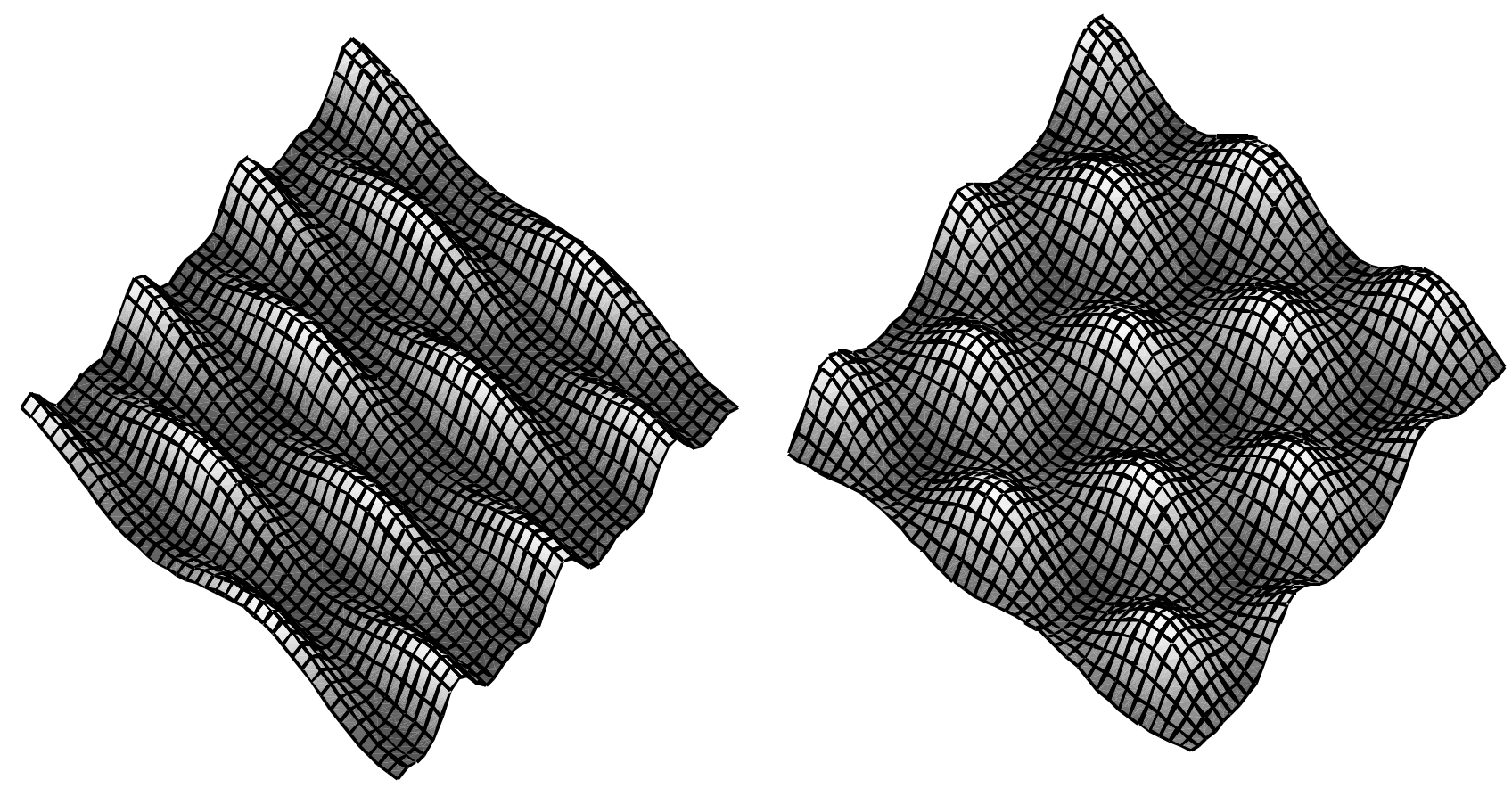

Figure 16. Examples of bed forms: (left) $\Delta \phi=\pi / 2, k_{x} / k_{y}=4$, and $a_{1}=a_{2}$ (brick pattern) and (right) $\Delta \phi=2 \pi / 3, k_{x} / k_{y}=2$, and $a_{1}=a_{2} / 2$ (hexagonal pattern). 
[45] We also performed a weakly nonlinear analysis considering the interaction of a triad of unstable modes. We studied in particular the resonant coupling between one longitudinal mode and two symmetrical modes and show that it can give birth to steady three-dimensional sand ripples that migrate in the flow direction. We obtain a large variety of 3-D ripples, ranging from brick patterns to hexagonal ones [Roos and Blondeaux, 2001].

[46] The situation we analyzed is very simplified and cannot be directly applied to natural situations where the flow is often turbulent. Nevertheless our study proves that it is not necessary to invoke the turbulence to explain the emergence of three-dimensional patterns. Moreover, this configuration allows us to have direct insights in the complex coupling between the fluid flow, the bed form morphology and the sand transport. A similar mechanism of nonlinear coupling between resonant modes is expected to occur even in a turbulent fluid flow. For instance, it is possible to perform the same type of calculation using the velocity field calculated by Hunt et al. [1988] in the turbulent case. A linear analysis shows that the "turbulent" marginal stability curve in the $\left(k_{x}, k_{y}\right)$ space has the same shape as that shown on Figure 4. Assuming that the pertinent nonlinearities come from the sand transport and not from the fluid motion, a nonlinear analysis should lead to the same conclusion as in the laminar case, that is, 3-D patterns can emerge because of a resonant coupling between a triad of unstable modes. Of course, one can expect the turbulent fluctuations to play an important role when they become as large as the mean value of the velocity field. The presence of recirculation regions in the flow would also invalidate the analysis on the basis of a matching asymptotic expansion. In this cases, the specific effects of the turbulence should be studied independently.

[47] Nevertheless, even in the laminar case, additional efforts are needed to predict the long-term evolution of three-dimensional sand ripple patterns. In particular, it would be necessary to perform a full nonlinear analysis to determine which three-dimensional patterns are stable and which parameters control their final shape. Furthermore, these theoretical predictions could be in principle tested in water flume experiments. Finally, the possible emergence of complex three-dimensional bed forms under a simple laminar and steady shear flow shows that the threedimensionality must be taken into account even in natural flows over erodible beds.

\section{Appendix A: Derivation of the Velocity Field}

[48] In this appendix, we present the derivation of the components $U_{1}$ and $V_{1}$ of the perturbed flow. Combining equations (17) and (18), we get

$$
\left[v\left(\partial_{z}^{2}-k^{2}\right)-i k_{x} \gamma z\right] \phi_{1}=\gamma k_{y} W_{1},
$$

where $\phi_{1}=k_{y} U_{1}-k_{x} V_{1}$. The solution for $\phi_{1}$ can be again expressed in terms of the Airy functions and of $W_{1}$ :

$$
\begin{aligned}
\phi_{1}(z)= & a_{3} A i\left(z^{\prime}\right)+b_{3} B i\left(z^{\prime}\right)+\pi l_{v}^{-2 / 3} e^{-i \pi / 6} \frac{k_{y}}{k_{x}^{1 / 3}} \\
& \cdot\left[B i\left(z^{\prime}\right) \int_{0}^{z} W_{1}(\zeta) A i\left(\zeta^{\prime}\right) \mathrm{d} \zeta-A i\left(z^{\prime}\right) \int_{0}^{z} W_{1}(\zeta) B i\left(\zeta^{\prime}\right) \mathrm{d} \zeta\right],
\end{aligned}
$$

with $z^{\prime}=e^{i \pi / 6}\left(k_{x} l_{v}\right)^{-2 / 3}\left(k_{x} z-i k^{2} l_{\nu}^{2}\right) . a_{3}$ and $b_{3}$ are integration constants. Finally, the use of the incompressibility equation (20) allows the derivation of explicit expressions for $U_{1}$ and $V_{1}$ :

$$
\begin{aligned}
& U_{1}(z)=\frac{1}{i k^{2}}\left(i k_{y} \phi_{1}(z)-k_{x} \partial_{z} W_{1}(z)\right) \\
& V_{1}(z)=\frac{1}{i k^{2}}\left(i k_{x} \phi_{1}(z)-k_{y} \partial_{z} W_{1}(z)\right) .
\end{aligned}
$$

\section{Appendix B: Expressions of the Shear Stress in the Long Wavelength Limit}

[49] We give here the asymptotic development of the perturbed bed shear stress in the long-wavelength limit $\left(k l_{\nu} \ll 1\right)$.

$$
\begin{aligned}
\frac{\tau_{b_{1 x}}}{h_{1} \tau_{b_{0}}}= & e^{i \pi / 6} l_{\nu}^{-2 / 3} k_{x}^{1 / 3} \frac{1.07 k_{x}^{2}-0.73 k_{y}^{2}}{k_{x}^{2}+k_{y}^{2}}+0.83 \frac{k_{x}^{2}}{\sqrt{k_{x}^{2}+k_{y}^{2}}} \\
& +e^{-i \pi / 6} l_{\nu}^{2 / 3} k_{x}^{-1 / 3}\left(0.43 k_{x}^{2}-0.53 k_{y}^{2}\right)+O\left(\left(k l_{\nu}\right)^{7 / 3}\right) \\
\frac{\tau_{b_{1 y}}}{h_{1} \tau_{b_{0}}}= & -0.34 e^{i \pi / 6} l_{\nu}^{-2 / 3} \frac{k_{x}^{4 / 3} k_{y}}{k_{x}^{2}+k_{y}^{2}}-0.83 \frac{k_{x} k_{y}}{\sqrt{k_{x}^{2}+k_{y}^{2}}} \\
& +0.96 e^{-i \pi / 6} l_{\nu}^{2 / 3} k_{x}^{2 / 3} k_{y}+O\left(\left(k l_{\nu}\right)^{7 / 3}\right)
\end{aligned}
$$

where $\tau_{b_{0}}=\eta \gamma$.

\section{Appendix C: Coefficients of the Nonlinear Analysis}

[50] The nonlinear coefficients of equations (38), (39), and (40) can be expressed as functions of the perturbed bed shear stress and read:

$$
\begin{aligned}
K_{1}= & -\frac{3 i}{4} k_{x} \Theta_{c_{0}}^{3 / 2} \mu^{-1 / 2}\left[(1+\mu)^{2} \frac{\tau_{2} \tau_{3}}{\sigma_{0}^{2}}-i k_{x} \frac{(1+\mu)}{\mu_{s}} \frac{\tau_{2}}{\sigma_{0}}-\frac{k_{x}^{2}}{4 \mu_{s}^{2}}\right], \\
K_{2,3}= & -\frac{3 i}{8} k_{x} \Theta_{c_{0}}^{3 / 2} \mu^{-1 / 2} \\
& \cdot\left[(1+\mu)^{2} \frac{\tau_{1} \tau_{3,2}^{*}}{\sigma_{0}^{2}}+\frac{k_{x}^{2}}{2 \mu_{s}^{2}}-i(1+\mu) \frac{k_{x}}{\mu_{s}}\left(\frac{\tau_{3,2}^{*}}{\sigma_{0}}-\frac{\tau_{1}}{2 \sigma_{0}}\right)\right] .
\end{aligned}
$$

[51] We have introduced the notations $\omega_{i}=\omega\left(\mathbf{k}_{i}\right)$ and $\tau_{i}=$ $\tau_{b_{1} x}\left(\mathbf{k}_{i}\right) / \tau_{b_{0}}\left(\omega\left(\mathbf{k}_{i}\right)\right.$ and $\tau_{b_{1} x}\left(\mathbf{k}_{i}\right)$ are given by equations (26), (27), and (24), respectively).

[52] The real coupling coefficients introduced in equations (41), (42), and (43) can be expressed as functions of $K_{1}, K_{2}, K_{3}$ :

$$
\begin{gathered}
\kappa_{1}=\Re\left(K_{1}\right) \cos (\Delta \phi)-\Im\left(K_{1}\right) \sin (\Delta \phi) \\
\kappa_{2}=\Re\left(K_{1}\right) \sin (\Delta \phi)+\Im\left(K_{1}\right) \cos (\Delta \phi) \\
\kappa_{3}=-2 \Re\left(K_{2}\right) a_{1}-\Re\left(K_{1}\right) \frac{a_{2}^{2}}{a_{1}} \\
\kappa_{4}=2 \Im\left(K_{2}\right) a_{1}-\Im\left(K_{1}\right) \frac{a_{2}^{2}}{a_{1}},
\end{gathered}
$$

with $\Delta \phi=2 \phi_{2}-\phi_{1}$. 


\section{References}

Abramovitz, M., and I. Stegun (1965), Handbook of Mathematical Functions, Dover, Mineola, N. Y.

Andreotti, B., P. Claudin, and S. Douady (2002), Selection of dune shapes and velocities part 2: A two-dimensional modelling, Eur. Phys. J. B, 28 $341-352$.

Bagnold, R. (1956), The flow of cohesionless grains in fluids, Philos. Trans. R. Soc. London, Ser. A, 249, 235-297.

Blondeaux, P. (1990), Sand ripples under sea waves. I: Ripple formation, J. Fluid Mech., 218, 1-17.

Carling, P., E. Gölz, H. Orr, and A. Radecki-Pawlik (2000), The morphodynamics of fluvial sand dunes in the river Rhine, near Mainz, Germany, Sedimentology, 47, 227-252.

Charru, F., H. Mouilleron, and O. Eiff (2004), Erosion and deposition of particles on a bed sheared by a viscous flow, J. Fluid Mech., 519, 55-80. Fredsoe, J., and R. Deigaard (1992), Mechanics of Coastal Sediment Transport, World Sci., Hackensack, N. J.

Hunt, J., S. Leibovich, and K. Richards (1988), Turbulent shear flow over hills, O. J. R. Meteorol. Soc., 114, 1435-1470.

Kovacs, A., and G. Parker (1994), A new vectorial bedload formulation and its application to the time evolution of straight river channels, J. Fluid Mech., 267, 153-183.

Kuru, W., D. Leighton, and M. McReady (1995), Formation of waves on a horizontal erodible bed of particles, Int. J. Multiphase Flow, 21, 11231140 .

Langlois, V., and A. Valance (2005), Two-dimensional sand ripples under continuous laminar shear flow, Phys. Rev. Lett., 94, 248001.

Loiseleux, T., D. Doppler, P. Gondret, and M. Rabaud (2004), Incipient grain transport and pattern formation at a sand surface sheared by a continuous laminar flow (part I), in Proceedings of the Second International Workshop on Marine Sandwaves and River Dune Dynamics, edited by S. J. M. H. Hulscher, T. Garlan, and D. Idier, pp. 200-207, Univ. of Twente, Enschede, Netherlands.
Maddux, T. B., J. M. Nelson, and S. R. McLean (2003a), Turbulent flow over three-dimensional dunes: 1 . Free surface and flow response, J. Geophys. Res., 108(F1), 6009, doi:10.1029/2003JF000017.

Maddux, T. B., S. R. McLean, and J. M. Nelson (2003b), Turbulent flow over three-dimensional dunes: 2. Fluid and bed stresses, J. Geophys. Res., 108(F1), 6010, doi:10.1029/2003JF000018.

McLean, S. (1990), The stability of ripples and dunes, Earth Sci. Rev., 29, $31-144$.

Nelson, J. (1990), The initial instability and finite-amplitude stability of alternate bars in straight channels, Earth Sci. Rev., 29, 97-115.

Raudkivi, A. (1997), Ripples on stream bed, J. Hydrol. Eng., 123, 58-64. Richards, K. (1980), The formation of ripples and dunes on an erodible bed, J. Fluid Mech., 99, 597-618.

Roos, P., and P. Blondeaux (2001), Sand ripples under sea waves. IV: Tile ripple formation, J. Fluid Mech., 447, 227-246.

Sauermann, G., K. Kroy, and H. Hermann (2001), Continuum saltation model for sand dunes, Phys. Rev. E, 64, 031305.

Valance, A., and V. Langlois (2005), Ripple formation over a sand bed submitted to a laminar shear flow, Eur. Phys. J. B, 43, 283-294.

Vittori, G., and P. Blondeaux (1992), Sand ripples under sea waves. III: Brick-pattern ripple formation, J. Fluid Mech., 239, 23-45.

Wilbers, A., and W. T. Brinke (2003), The response of subaqueous dunes to floods in sand and gravel bed reaches of the Dutch Rhine, Sedimentology, $50,1013-1034$

V. Langlois, Groupe Matière Condensée et Matériaux, CNRS, Université de Rennes, Bât. 11A, F-35000 Rennes cedex, France. (vincent.langlois@ univ-rennes1.fr)

A. Valance, Groupe Matière Condensée et Matériaux, CNRS, Université de Rennes, Bât. 11A, F-35042 Rennes cedex, France. (alexandre.valance@ univ-rennes1.fr) 